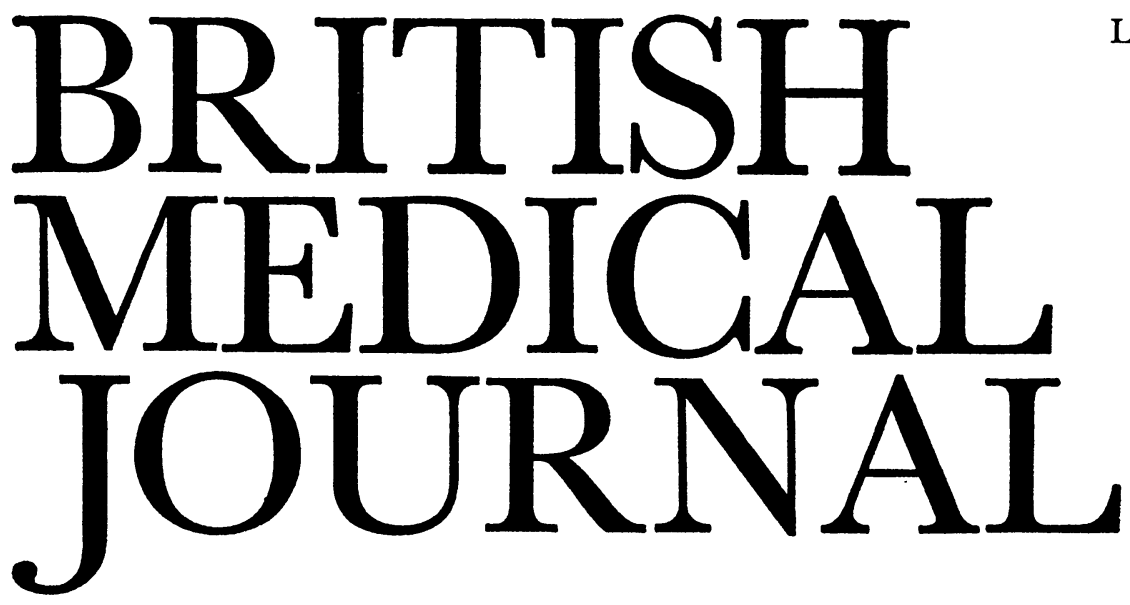

\title{
Massive Pulmonary Haemorrhage in the Newborn
}

Imminent death is generally feared for a young baby ill with respiratory distress, infection, or a surgical condition who shows signs of circulatory failure with slowing of the pulse, blotchy discoloration of the skin, and sclerema, followed by bleeding into the skin or from the respiratory or alimentary passages. At necropsy both new and old sites of bleeding can usually be found in the paraventricular areas of the brain, the lower oesphagus or duodenum, within the viscera such as the adrenals, or within the lungs. As these lesions are usually identified with ease and are often the only lesions found with the naked eye, death is commonly ascribed to them. Thus many deaths have been recorded as due to "acute haemorrhagic pneumonia."

Pathologists dealing with many newborn babies are often aware of the different patterns of lesions found in cases of neonatal death from different hospitals. The "haemorrhagic" infant tends to come from a "high-powered" unit, and it has always been something of a question whether to attribute this to over-enthusiastic resuscitation or to prolonged survival allowing the baby to reach a later terminal state of disease.

C. B. Bcothby and D. J. deSa, ${ }^{1}$ in the Oxford area, noted that an increased incidence of massive pulmonary haemorrhage as a cause of death coincided with a falling perinatal mortality rate during 1969-71. They attributed this to the effects of oxygen toxicity on the lung. Workers at the pulmonary unit at University College Hospital, London, have attempted to analyse factors leading to massive pulmonary haemorrhage. ${ }^{2}$ They describe 15 infants, all of whom were severely ill; 11 required endotracheal intubation at birth, six had respiratory distress syndrome, and three haemolytic disease. All but eight were on a respirator at the onset of the new pulmonary symptoms.

The average age of the babies was 37 hours, though the range was from 20 minutes to 14 days. First there were periods of slow, gasping breathing or of apnoea. These accompanied attacks of bradycardia and peripheral vasoconstriction. Bradycardia was seen to precede the bleeding state in several infants. This was followed by the appearance of blood-stained fluid in the endotracheal tubes or at the larynx. The fluid usually appeared to be blood, or fluid tinged with blood; in one child it started as a clear fluid, and later became blood-stained. The outpouring continued for several minutes; then if the child was resuscitated it ceased, though in five infants it recurred. All of the children died at an average of five hours later.

The U.C.H. workers collected the lung effusion from 12 infants and at the same time took peripheral blood from 11 of them. The amount of blood in the effusion varied from 0 to $94 \%$. The concentrations of electrolytes, urea, and phosphatase differed in no significant way from those in the blood plasma. They estimated the plasma proteins of different molecular size in the effusion and in the circulating plasma, and, after correction for the diluting effect of the blood in the pulmonary effusion, showed that there was sieving out of the smaller blood proteins from the plasma. Disseminated intravascular coagulation was detected in one child before bleeding occurred, and in five out of ten a state suggestive of this appeared later. Seven showed no evidence of disseminated intravascular coagulation at any stage. The necropsy findings seemed to be peculiarly uninformative. One of the 13 examined had a subarachnoid haemorrhage and five had intraventricular haemorrhages. The lung effusion consisted of plasma transudate with variable amounts of blood - that is, haemorrhagic oedema fluid-and these workers postulate that the lungs must have developed pores of between 200 and $300 \AA$ radius. They conclude that massive pulmonary haemorrhage is due to left-sided ventricular failure producing an increase in alveolar capillary pressure. They do not consider that disseminated intravascular coagulation is an important initiating factor.

The data are at present too limited to allow a common cause for this condition to be assumed. Some of these infants show changes in the heart that would support the concept of a left-sided heart failure, but not all do so. Experimentally, massive pulmonary haemorrhage can be produced in a variety of ways. Examples are damage to the alveolar epithelium; damage to the capillary endothelium, as in inhalation of oxygen at high concentrations; mechanical damage, as in mechanical overventilation of a lung, with peripheral alveolar collapse; and excessive blood transfusion.

In perinatal deaths massive pulmonary haemorrhage occurs in several forms. Sometimes it is part of the respiratory-distress and hyaline-membrane picture, or oxygen intoxication with intra-alveolar haemorrhage; sometimes the haemorrhage is almost exclusively interstitial; occasionally it is almost wholly intralymphatic. Ante-mortem pulmonary venous thrombosis is a fairly common accompaniment of 
haemorrhage and easily missed at necropsy. Regurgitation with inhalation can sometimes be a precipitating factor. Massive pulmonary haemorrhage can be produced in newborn babies accidentally by catheters being passed too far through endotracheal tubes.

Massive pulmonary haemorrhage is a condition that can be anticipated in severely ill newborn babies, to which possibly preventable factors may contribute. It deserves thorough investigation, for it represents a barrier to the control of neonatal mortality.

\footnotetext{
1 Boothby, C. B., and deSa, D. J., Archives of Disease in Childhood, 1973,

2 Cole, V., Normand, I. C. S., Reynolds, E. O. R., and Rivers, R. P. A., Pediatric:; $1973,51,175$.
}

\section{Future of a London School}

Next year will be the 75th year of the London School of Hygiene and Tropical Medicine as a school of tropical medicine and its 50th year as a school of public health. During the last two years it has been reviewing its present position and future role in this rapidly changing world, both at home and overseas.

The school is unique, at least in Great Britain, in having within one institution the balanced array of disciplines and experience necessary for teaching and research over the whole field of preventive medicine, whether in industrialized or developing countries. Latterly it has given more emphasis to epidemiology and the sociological and management aspects of health services. Now the context is changing fast. In Britain the teaching of community or social medicine is growing in strength in many (if not all) undergraduate schools. The needs of developing countries, particularly the wealthier ones, will increasingly resemble those of the industrialized countries in preventive and social medicine, though communicable disease will continue to pose larger problems in them. The corollary is that less advanced courses in tropical medicine and hygiene should be progressively taken over locally or regionally and the school's resources transferred to more advanced teaching.

Meanwhile, in response to the needs of the reorganized National Health Service for some 1,000 community physicians and for an annual intake of 30-40 each year, the school proposes to build up its M.Sc. course in social medicine to twice its present size provided it can obtain funds and staff. The training of specialists in community medicine, the school believes, should include at least four years' training with both vocational and in-service components. In addition the M.Sc. in medical statistics will play a major part in the training of non-medical (and some medical) graduates concerned with the technical aspects and detailed planning of information services. The school is also considering its role (both in teaching and research) in environmental medicine including toxicology, and it is clear that an adequate range of high quality teaching can be provided only by the pooling of academic resources within the university.

As there is not much room for further expansion on its present site the school is looking elsewhere. Its activities in the service of students of more than 80 nationalities have been expanding at an increasing rate lately. Work at the Winches Farm Field Station in Hertfordshire continues to grow; a clinical nutrition and metabolism unit has been built at the Hospital for Tropical Diseases; and premises have been leased near the school in Bedford Square for a centre for extension training in community medicine and for a 'unit to study the control of chronic diseases.

The Royal Commission on Medical Education ${ }^{1}$ recommended the merging of the school with University College and the Royal Free Hospital in a new teaching group, but the school somewhat naturally is resisting the proposal, for its functions have little in common with those of the other institutions. At present it is an independent school within London University. If it is to frame its policy according to its special needs it must remain for all practical purposes self-governing and financially autonomous. However, the type of association recommended by the Royal Commission is being examined in terms of two undergraduate complexes, and the merits of a possible association with Northwick Park Hospital and the Medical Research Council's Clinical Research Centre are being studied also. The school hopes to define its long-term objectives in the early autumn and then to decide in principle about its location before the end of the year.

1 Royal Commission on Medical Education 1965-68, Report. London, H.M.S.O., 1968.

\section{Suction Drainage in Breast Surgery}

Ever since William Halsted ${ }^{1}$ first carried out a radical mastectomy at the Johns Hopkins Hospital in 1882 surgeons have been concerned about the problems of wound healing of the resultant extensive defects and skin flaps. In his original cases Halsted fashioned a flap to cover the axillary contents and then allowed the large remaining wound to heal by granulation tissue. But soon primary closure of the defect by suture or skin grafting became the invariable practice. The problems which faced the early surgeons remain with us today-namely, necrosis of the skin flaps, breakdown of the wound, haematoma, and serous collection ("seroma") under the flap with consequent wound infection. As well as delaying the patient's discharge from hospital the consequences of these complications include an ugly scar, tethering of the skin to the chest wall, limitation of shoulder movement, and development of unpleasant and even disabling lymphoedema of the arm.

To obliterate the dead space between the skin flaps and the chest wall the standard practice was, and in many centres remains, the insertion of one or more tube drains combined with a voluminous gauze dressing held firmly in place by careful bandaging or strapping against the thoracic wall. In 1951 T. G. Orr ${ }^{2}$ introduced tension sutures tied over rubber tubing to tack the flaps to the underlying intercostal muscles and latissimus dorsi; a single drain was used. E. L. Keyes and his colleagues ${ }^{3}$ used silk stitches to hold the flaps against the chest wall and inserted up to five soft (Penrose) drains. They reported that in their 17 cases there was less sloughing and quicker healing than in a similar number treated on conventional lines. B. B. Larsen and C. Hugan ${ }^{4}$ tacked down the skin flaps with 30 to 50 subcutaneous cotton sutures and avoided a drain if possible. The extent of the problem is shown by their figures. Of their 30 patients eight required one or more aspirations of fluid colleation, one developed an infected haematoma, and four had wound sloughing, while 